\title{
Interventions for reducing benzodiazepine use in older people: meta-analysis of randomised controlled trials
}

Rebecca L. Gould, Mark C. Coulson, Natasha Patel, Elizabeth Highton-Williamson

and Robert J. Howard

\section{Background}

The use of benzodiazepines has been advised against in older people, but prevalence rates remain high.

\section{Aims}

To review the evidence for interventions aimed at reducing benzodiazepine use in older people.

\section{Method}

We conducted a systematic review, assessment of risk of bias and meta-analyses of randomised controlled trials of benzodiazepine withdrawal and prescribing interventions.

\section{Results}

Ten withdrawal and eight prescribing studies met the inclusion criteria. At post-intervention, significantly higher odds of not using benzodiazepines were found with supervised withdrawal with psychotherapy (odds ratio $(\mathrm{OR})=5.06,95 \% \mathrm{Cl} 2.68-9.57, P<0.00001)$ and withdrawal with prescribing interventions $(\mathrm{OR}=1.43,95 \% \mathrm{Cl} 1.02-2.02$, $P=0.04$ ) in comparison with the control interventions treatment as usual (TAU), education placebo, withdrawal with or without drug placebo, or psychotherapy alone. Significantly higher odds of not using benzodiazepines were also found for multifaceted prescribing interventions $(\mathrm{OR}=1.37,95 \% \mathrm{Cl} 1.10-1.72, P=0.006)$ in comparison with control interventions (TAU and prescribing placebo).

\section{Conclusions}

Supervised benzodiazepine withdrawal augmented with psychotherapy should be considered in older people, although pragmatic reasons may necessitate consideration of other strategies such as medication review.

\section{Declaration of interest}

None.
The long-term use of benzodiazepines has been advised against in older people owing to adverse outcomes, ${ }^{1-4}$ including increased risks of cognitive impairment, falls, fractures, traffic accidents, delirium and dependence. ${ }^{5-10}$ The American Geriatrics Society 2012 Beers Criteria Update Expert Panel has recommended avoidance of benzodiazepines for insomnia, agitation or delirium in older people owing to slower metabolism of and increased sensitivity to such medications. ${ }^{11}$ Despite previous recommendations, estimates of the prevalence of benzodiazepine use in older people remain high. Estimates from recent studies range from $12 \%$ to $32 \%,{ }^{12,13}$ with prevalence rates being even higher in those with psychiatric disorders. ${ }^{14,15}$ Benzodiazepine rates of $57-59 \%$ have been reported in the latter group, ${ }^{14}$ and more specifically in those with depression or anxiety disorder. ${ }^{15}$ Benzodiazepine prevalence rates may continue to be high for numerous reasons. These include a lack of specialist knowledge about benzodiazepine prescribing in geriatric care; ${ }^{14}$ difficulties in translating prescribing guidelines into clinical practice, including a perceived lack of alternative evidence-based treatments and unwillingness of older people to discontinue benzodiazepines ${ }^{16}$ a perceived lack of priority for physicians (e.g. due to greater physical health needs); and physiological and psychological dependency issues. Consequently, interventions that aim to reduce benzodiazepine use by helping patients to withdraw from them or by changing prescribing (and hence benzodiazepine use) may help to reduce prevalence rates in older people.

A stepped care approach has been proposed for withdrawal from benzodiazepines, comprising minimal interventions such as advisory letters or consultations with healthcare professionals, supervised gradual withdrawal and specialised care (e.g. supervised withdrawal augmented with psychotherapy or pharmacotherapy). ${ }^{17}$ These proposals have been supported by meta-analyses and systematic reviews of interventions that included adults of any age, ${ }^{18-21}$ but have not been examined specifically in older people. Meta-analyses have also shown that this stepped care approach may not be applicable in all settings. For example, gradual withdrawal plus pharmacotherapy was found to be superior to gradual withdrawal alone for participants using benzodiazepines recruited from in-patient and out-patient settings, ${ }^{20}$ but not when limited to general practice and outpatient settings. ${ }^{18}$ With respect to strategies for changing prescribing (and hence drug use) in older people, a number of interventions have been suggested. ${ }^{22-25}$ These include completing regular medication reviews and consultations with patients (which overlap with minimal withdrawal interventions noted above), providing educational outreach programmes to prescribers, conducting audits and providing feedback on prescribing patterns, implementing electronic prescribing alerts and providing patient support groups. Previous meta-analyses and reviews provide support for these strategies, ${ }^{23-27}$ but were not focused specifically on benzodiazepines (focusing primarily on inappropriate prescribing in care homes) $)^{23,24,26,27}$ and/or examined interventions in a wider age group. $^{25,27}$

The extent to which findings from withdrawal and prescribing studies specifically apply to older people is uncertain as the majority of studies used a broad age range. Certainly, inconsistencies exist in the literature with respect to the influence of age on benzodiazepine-related outcomes: for example, some studies reported more favourable outcomes for older than younger people, ${ }^{28-31}$ whereas other studies reported less favourable outcomes. $^{32-35}$ Yet other studies reported no effect of age, ${ }^{36-42}$ although the degree to which some of them were sufficiently powered to detect an effect is unclear owing to failures to report age ranges. ${ }^{36,37,41,42}$

It may be that withdrawal interventions are more successful in older people because of age-related physiological processes (e.g. 
slower metabolism of benzodiazepines), which may result in slower clearance rates from the body. ${ }^{28}$ This may lead to less severe withdrawal symptoms, ${ }^{28,31}$ or at least a different presentation in symptoms (e.g. disorientation and confusion rather than anxiety or insomnia). ${ }^{43}$ On the other hand, it may be that the effectiveness of interventions is reduced in older people owing to long-term dependency issues such as lack of motivation to reduce medication. ${ }^{10,44}$ Furthermore, withdrawal interventions involving psychotherapy might be less effective in older people owing to difficulties in addressing chronic underlying disorders such as anxiety or insomnia. Certainly, smaller effect sizes in favour of cognitive-behavioural therapy (CBT) for generalised anxiety disorder have been reported in older participants compared with younger people. ${ }^{45}$ Alternatively, an interaction of these factors might mean that interventions are as efficacious in older people as in wider populations. Thus, there is a need to clarify the effectiveness of interventions for reducing benzodiazepine use specifically in older people. Consequently, our aims were: first, to conduct a systematic and critical review of the evidence from randomised controlled trials (RCTs) targeting the reduction of benzodiazepine use in older people in a variety of settings (in-patient, out-patient, community and care homes); second, to compare the efficacy of different types of interventions for reducing benzodiazepine use; and third, to examine the persistence of beneficial intervention effects at short-term (0.5-3 months) and longer-term (12 months) follow-up.

\section{Method}

Medline, EMBASE, PsycINFO, Web of Knowledge and the Cochrane Collaboration Central Register of Controlled Trials were searched on 6 October 2012 using the following terms: [benzodiaz ${ }^{\star}$ OR hypnotic ${ }^{\star}$ OR anxiolytic ${ }^{\star}$ OR psychotropic ${ }^{\star}$ OR sedative $^{\star}$ OR tranquil ${ }^{\star}$ OR alprazolam OR bromazepam OR clobazam OR clonazepam OR diazepam OR flunitrazepam OR lorazepam OR midazolam OR nitrazepam OR oxazepam OR temazepam OR triazolam OR zolpidem OR zopiclone] AND [taper ${ }^{\star}$ OR discontinu* OR reduc* OR withdraw ${ }^{*}$ OR cessation OR long-term OR chronic OR dependen ${ }^{\star}$ OR overuse OR misuse OR addiction OR abuse OR prescri ${ }^{\star}$ ] AND [older OR elder ${ }^{\star}$ OR geriatr* OR "old age" OR "late life" OR late-life] AND [RCT OR random ${ }^{\star}$ ]. Studies were also identified from citations in studies, reviews and meta-analyses of interventions that aimed to reduce benzodiazepine use in adults of any age.

\section{Inclusion and exclusion criteria}

Studies were included if they met the following criteria:

(a) the study was a peer-reviewed parallel RCT, cluster RCT (with more than two clusters) or crossover RCT (with data available for separate crossover periods);

(b) a primary or secondary aim was to reduce benzodiazepine use by withdrawal interventions or interventions for changing benzodiazepine prescribing;

(c) the intervention was compared with a non-active control such as waiting list or treatment as usual (TAU), or with an active control (e.g. pill placebo, social support/talking/education placebo, or psychotherapy alone);

(d) the mean, median or modal age of participants in the study was 60 years or more, with the minimum age of participants being 50 years.

The age criteria were chosen to reflect official definitions of 'older people, ${ }^{, 6,47}$ and because higher prevalence rates of benzodiazepine use have been reported in this age group. ${ }^{48}$ Studies that involved both older and working-age people were included if age-specific analyses were reported. Studies that failed to report mean or minimum ages were included only if the setting was exclusively for older people, such as a residential care home. Studies were excluded if data were insufficient to permit the calculation of effect sizes, or if the number of participants in each condition was fewer than five.

\section{Study selection and assessment of trial quality}

Studies were independently screened and selected if they were considered to meet inclusion criteria by three authors (R.L.G., N.P. and M.C.C.). Study quality in five areas of bias known to affect clinical outcomes (sequence generation, allocation concealment, masking of outcome assessors, incomplete outcome data and selective outcome reporting) was assessed using a risk of bias tool recommended by the Cochrane Collaboration. ${ }^{49}$ The control of bias in each area (i.e. whether it was adequate, partially adequate or inadequate/unclear) was independently, blindly rated by the same three authors for each study. Discussion and consensus were used to resolve any discrepancies.

\section{Outcome measures}

The main outcome measure was odds ratio in relation to not using benzodiazepines, at the level of patients or prescriptions. Data were examined at the post-intervention assessment and at short-term (0.5-3 months) and longer-term (12 months) follow-up. Following a previous meta-analysis, ${ }^{18}$ wherever possible a conservative approach was taken whereby all patients withdrawing from the study were assumed to have continued using benzodiazepines (akin to intention-to-treat rather than completer analyses). Data were extracted from each study by three authors (R.L.G., N.P. and M.C.C.), study authors were contacted for further information if necessary, and any discrepancies were resolved through discussion and consensus.

\section{Calculation of effect sizes}

Odds ratios with $95 \%$ confidence intervals were calculated for each study. These were log-transformed for input into metaanalyses and back-transformed for reporting purposes. If a study included two or more comparator conditions then the experimental and control conditions were combined in order to avoid making unit-of-analysis errors. ${ }^{50}$ Data from cluster RCTs were only included with data from parallel RCTs if there was adjustment for clustering at the individual level. ${ }^{50}$ In cases where there was no adjustment, this was calculated using study-specific estimates of the design effect or variance inflation factor. ${ }^{51}$ The design effect was calculated as $1+(m-1) p$, where $m$ is the average cluster size and $p$ is the sample estimate of the intracluster correlation coefficient (ICC). If an ICC was not reported, this was taken from a similar study and used to calculate study-specific estimates of the design effect. Data from crossover RCTs were included with data from parallel RCTs only if data were available separately for all crossover periods, in addition to combined data from all crossover periods, thus enabling estimations of the risk of bias from carry-over effects. ${ }^{52}$

\section{Meta-analyses}

Random effects meta-analyses using a DerSimonian \& Laird estimator based on generic inverse variance weights were conducted using the metan function in Stata version 11.2 and RevMan version 5.1 on Windows OS. ${ }^{53,54}$ Separate groups of 
meta-analyses were conducted for withdrawal and prescribing studies owing to anticipated heterogeneity in interventions and control conditions. There was an overlap between some withdrawal and prescribing interventions (e.g. those involving the provision of advisory letters or consultations with a withdrawal plan), thus a study could potentially be included in both groups of meta-analyses. Following evidence of differential effectiveness of withdrawal interventions in adults of any age, ${ }^{18-21}$ separate meta-analyses were conducted for studies of:

(a) withdrawal with a prescribing intervention (i.e. an intervention that included a withdrawal plan with one or more prescribing interventions such as medication review, consultation or education);

(b) supervised withdrawal alone (i.e. supervised by study personnel or healthcare professionals);

(c) supervised withdrawal augmented with psychotherapy (e.g. CBT or psychological consulting);

(d) supervised withdrawal augmented with pharmacotherapy (e.g. non-benzodiazepine medication, another type of benzodiazepine or a pill placebo).

Separate meta-analyses were also conducted for single-faceted $v$. multifaceted interventions as the latter were reported to be more effective than the former. ${ }^{25}$ Single-faceted interventions were defined as those that included only one prescribing intervention (e.g. education alone). Multifaceted ones were those that included two or more prescribing interventions (e.g. education plus medication review). In addition, separate meta-analyses were conducted for different assessment periods (post-intervention, short-term and longer-term follow-up) in order to avoid nonindependence of effect sizes. Finally, sensitivity analyses were conducted in order to examine the robustness of findings when not adjusting for clustering at the individual level in cluster RCTs, using fixed effects rather than random effects analyses and excluding any study that received a rating of inadequate/unclear in all five areas of risk of bias.

Statistical significance of the estimated average treatment effect in each meta-analysis was examined using the $Z$-test. Percentage of variability in treatment effects between studies due to heterogeneity rather than sampling error or chance was assessed in each meta-analysis using Cochran's Q-test and the $I^{2}$ statistic. Values of $0 \%, 25 \%, 50 \%$ and $75 \%$ indicated no, low, moderate and high heterogeneity respectively. ${ }^{55}$ If there was evidence of low to high heterogeneity, $95 \%$ prediction intervals were calculated in order to provide an estimate of the range of treatment effects within an individual study setting. ${ }^{56}$ Publication bias was examined using funnel plots and Egger's regression asymmetry test. ${ }^{57}$ If publication bias was detected, a nonparametric trim and fill method was used to impute missing studies and re-estimate the pooled effect size. ${ }^{58}$ An alpha level of 0.05 was used for tests of the estimated average treatment effect and publication bias, whereas $\alpha=0.10$ was used for tests of heterogeneity due to reductions in sensitivity of Cochran's Q-test with small numbers of studies. Finally, the number needed to treat (NNT) to enable one additional person to not use benzodiazepines with the treatment rather than control intervention was calculated. This was computed from odds ratios, whereby the median control group risk across studies in the same group served as the assumed control risk. ${ }^{50}$

\section{Subgroup and meta-regression analyses}

A priori subgroup and meta-regression analyses were used to examine whether any between-study heterogeneity could be explained by type of intervention in withdrawal and prescribing studies. In addition, analyses were completed for two further variables (underlying pathology and setting) as recommended by an anonymous reviewer. Subgroup analyses in RevMan 5.1 indirectly assessed differences in overall effect sizes between subgroups using a test of heterogeneity. ${ }^{59}$ Bonferroni-corrected alpha levels were chosen to indicate statistically significant subgroup differences at each time point in order to control for the risk of false positives in multiple subgroup analyses. ${ }^{60}$ Random effects univariate meta-regression analyses formally examined whether moderating variables were associated with effect sizes. The $I^{2}$ statistic was used to measure the percentage of variability in treatment effects due to subgroup differences rather than sampling error or chance. Log-transformed odds ratios were entered into meta-regression analyses as the dependent variable, whereas dichotomous moderating variables were dummy-coded independent variables. Analyses were conducted using the metareg function in Stata version 11.2 with restricted maximum likelihood estimation and Knapp-Hartung adjustment. Any factor that was significant in univariate analyses was entered into a random effects multivariate meta-regression analysis, and a joint test of covariates was calculated in order to control for the risk of false positives. In addition, a Monte Carlo permutation test (with 1000 random permutations) was performed to adjust $P$-values for multiple comparisons. ${ }^{61}$ An alpha level of 0.05 was chosen to indicate statistical significance.

\section{Results}

The PRISMA checklist was used to guide reporting (see online Table DS1). ${ }^{62}$

\section{Identification and characteristics of included studies}

Literature searches identified 2848 studies, of which 16 met inclusion criteria (Fig. 1) ${ }^{63-79}$ Of these, ten focused on withdrawal from benzodiazepines, ${ }^{63-73}$ and eight focused on changing benzodiazepine prescribing (and hence benzodiazepine use)..$^{70,72-79}$ Two studies were included in both meta-analyses as the treatment intervention qualified as both a withdrawal and multifaceted prescribing intervention for some participants..$^{70,72,73}$ The characteristics of each of the included studies are described in online Table DS2.

\section{Withdrawal interventions}

For the withdrawal studies, supervised gradual withdrawal was the most common type of intervention (6 studies); 1 study employed supervised abrupt withdrawal, 1 study compared both gradual and abrupt withdrawal and 2 studies used gradual withdrawal with a prescribing intervention (for some participants only). Withdrawal was augmented with pharmacotherapy in 4 studies and with psychotherapy in 4 studies. No study compared withdrawal alone with a control condition. Psychotherapy was described as CBT in 2 studies, behavioural therapy (i.e. relaxation training) in 1 study and 'psychological consulting' in 1 study. Pharmacotherapy comprised melatonin (1 study), carbamazepine (1 study), pill placebo (2 studies) and low-dose lormetazepam (2 studies). The length of withdrawal interventions ranged from 1 week to 12 months. Studies employed a variety of comparison conditions: withdrawal alone (3 studies), withdrawal with pill placebo (3 studies), withdrawal with combined psychotherapy and pill placebo (1 study), psychotherapy alone (1 1 study) and an education control (1 study). Two studies compared withdrawal with a non-active control condition (TAU). The most common 


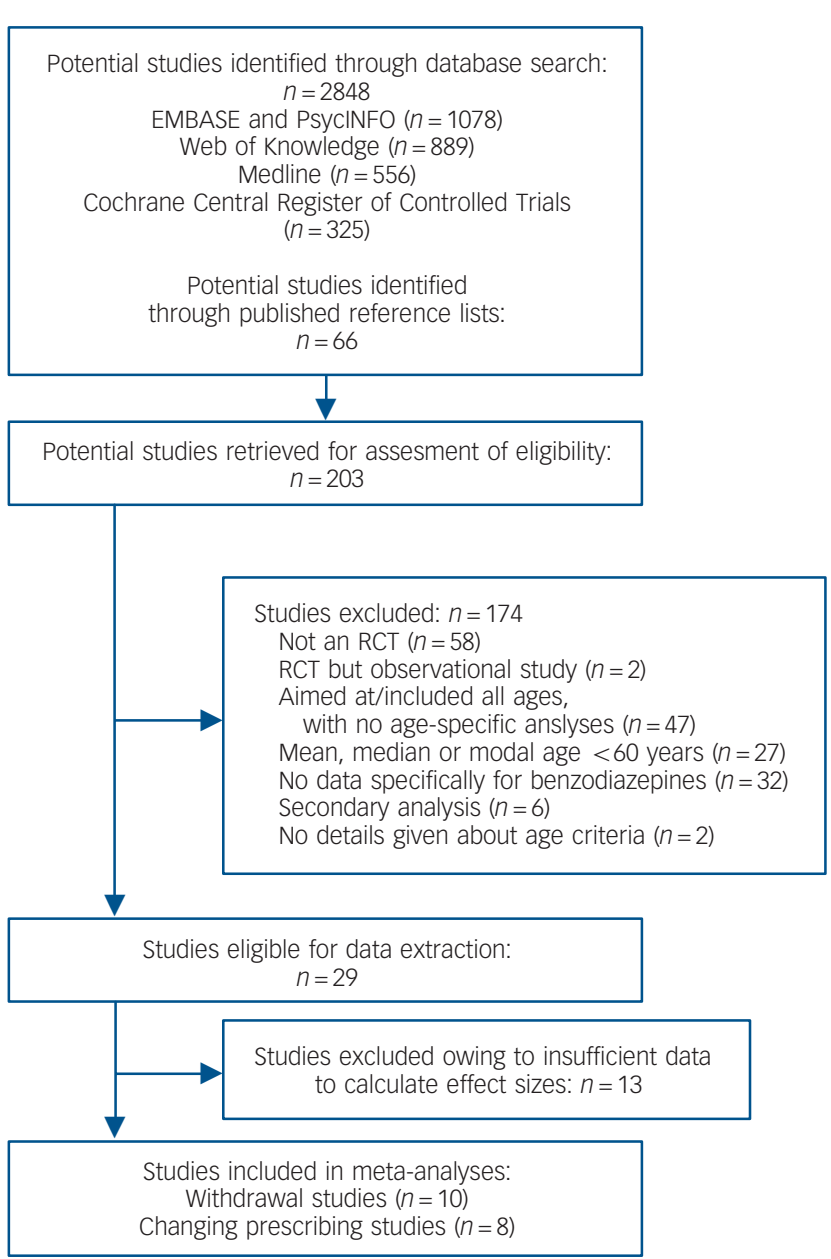

Fig. 1 Flow of studies from identification to meta-analyses. $\mathrm{RCT}$, randomised controlled trial.

setting of withdrawal interventions was the community (6 studies), followed by in-patient wards (2 studies) and out-patient clinics and care homes (1 study each). The majority of studies recruited participants who were using benzodiazepines for insomnia; in 5 studies this was for insomnia alone, in 2 studies for insomnia and/or anxiety and for anxiety alone in only 1 study. The most frequent inclusion criterion with respect to duration of benzodiazepine use was 3 months or more ( 3 studies), followed by 6 months ( 2 studies), then 1 month and 12 months (1 study each), with 3 studies not specifying duration. The mean age of participants was 74.1 years and the mean minimum age was 61.9 years. The mean percentage of female participants was $73.4 \%$. Follow-up data were reported in 5 studies, with $0.5-3$ months being the most frequent follow-up assessment period.

\section{Prescribing interventions}

The most common components of prescription interventions were education (6 studies), medication reviews ( 6 studies) and provision of prescribing feedback ( 3 studies). The length of interventions varied from 1 month to 12 months. Half of the studies employed a cluster RCT design (with the unit of randomisation typically being care homes). Non-active controls (TAU) were employed in the majority of prescribing studies (6 studies), with 2 studies using an active control. Half of the studies were conducted in care homes and half in the community. The majority of studies primarily aimed interventions at physicians and/or other staff ( 5 studies); 2 studies targeted patients and 1 involved both staff and patients. Only 1 prescribing study reported reasons for the use of benzodiazepines, and no study reported inclusion criteria for the duration of benzodiazepine use. The mean age of participants was 79.4 years and the mean minimum age was 65.8 years. The mean percentage of female participants was $77.4 \%$. Follow-up data with respect to the use of benzodiazepines were not reported in any prescribing study, although this was unclear in 2 studies and so the final follow-up assessment was used as the post-intervention assessment in these cases.

\section{Trial quality of included studies}

Potential sources of bias for each study are listed in online Table DS3. No study achieved adequate ratings in all areas of risk of bias, and no study was rated as inadequate/unclear in all five areas. The majority of benzodiazepine withdrawal studies were rated as having three or four inadequate/unclear areas of bias (7 out of 10 studies). In contrast, the majority of prescribing studies received only one or two inadequate/unclear ratings ( 5 out of 8 studies). Allocation concealment was the most inadequately/ unclearly addressed area of bias in withdrawal and prescribing studies, whereas incomplete data and selective reporting of data were the most adequately addressed areas of bias for both types of study.

\section{Meta-analysis: withdrawal interventions}

\section{odds of not using benzodiazepines}

The average effect of supervised withdrawal with psychotherapy at post-intervention was to make the odds of not using benzodiazepines 5.06 times higher than for control interventions (95\% CI 2.68-9.57, $P<0.00001, \mathrm{NNT}=3$; Fig. 2 and online Table DS4). In contrast, the odds were 1.43 times higher for withdrawal with a prescribing intervention (95\% CI 1.02-2.02, $P=0.04, \mathrm{NNT}=13)$ and 1.31 times higher for supervised withdrawal with pharmacotherapy (95\% CI $0.68-2.53, P=0.42$, NNT $=20)$. At $0.5-3$ months follow-up, on average, the odds of not using benzodiazepines were 3.90 times higher for supervised withdrawal with psychotherapy compared with control interventions (95\% CI 1.94-7.82, $P=0.0001$, NNT $=4$; Fig. 3 and Table DS4). However, there was moderate heterogeneity in effect sizes, although this was not significant $(36 \%, P=0.20)$. The $95 \%$ prediction intervals suggested that withdrawal with psychotherapy might not always be effective in individual settings in comparison with control interventions. Only one study examined supervised withdrawal with pharmacotherapy: here, the odds of not using benzodiazepines were 4.00 times higher than for the control intervention ( $95 \%$ CI $0.68-23.41, \mathrm{NNT}=5$ ). No study examined withdrawal with a prescribing intervention.

As can be seen in Fig. 4 and Table DS4, significantly higher odds of not using benzodiazepines were found with supervised withdrawal plus psychotherapy at 12 months follow-up. The average effect of treatment was to make the odds of not using benzodiazepines 3.00 times higher for supervised withdrawal plus psychotherapy compared with control interventions (95\% CI $1.43-6.28, P=0.004, \mathrm{NNT}=5)$. However, moderate heterogeneity in effect sizes was found, although this was not significant $(32.0 \%$, $P=0.23$ ). On average, although studies with psychotherapy appeared effective at aiding withdrawal from benzodiazepines in comparison with control interventions, 95\% prediction intervals suggested they might not always be effective in individual settings. No study assessed withdrawal with a prescribing intervention or supervised withdrawal with pharmacotherapy. 


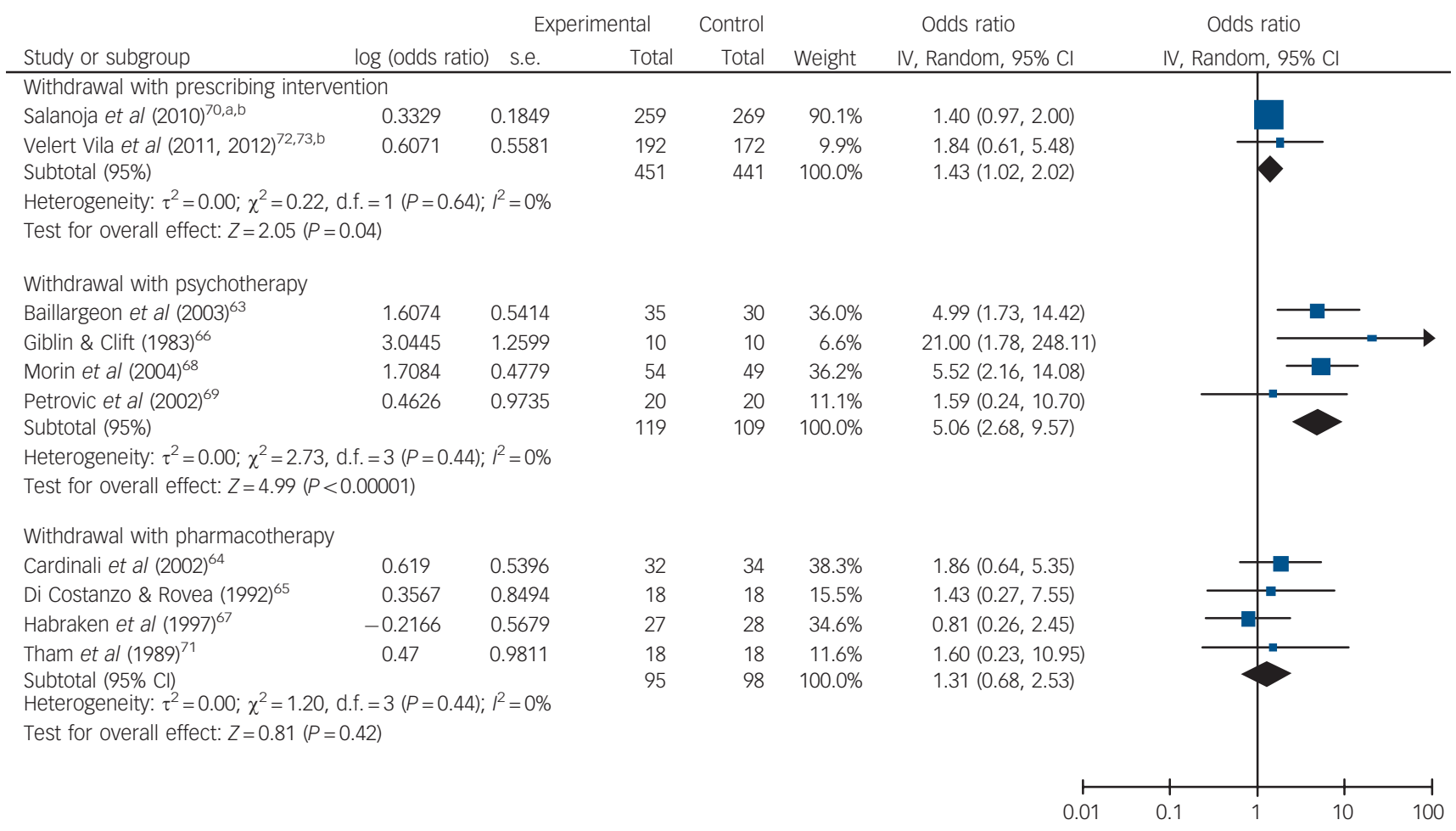

Fig. 2 Pooled odds ratios in relation to not using benzodiazepines in studies aimed at withdrawal from these drugs at post-intervention. a. Participants leaving the study were not assumed to have continued using benzodiazepines as it was not possible to calculate this. b. Withdrawal occurred only for some participants.

\begin{tabular}{|c|c|c|c|c|c|c|c|c|c|}
\hline \multirow[b]{2}{*}{ Study or subgroup } & \multicolumn{3}{|c|}{ Experimental } & \multicolumn{2}{|l|}{ Control } & Odds ratio & \multicolumn{2}{|c|}{ Odds ratio } & \\
\hline & $\log$ (odds ratio) & s.e. & Total & Total & Weight & IV, Random, 95\% Cl & \multicolumn{2}{|c|}{ IV, Random, 95\% Cl } & \\
\hline \multicolumn{9}{|l|}{ Withdrawal with psychotherapy } & \\
\hline Baillargeon et al $(2003)^{63}$ & 1.2192 & 0.5219 & 35 & 30 & $27.8 \%$ & $3.38(1.22,9.41)$ & & & \\
\hline Giblin \& Clift $(1983)^{66}$ & 2.6027 & 0.7136 & 30 & 30 & $18.2 \%$ & $13.50(3.33,54.67)$ & & & \\
\hline Morin et al $(2004)^{68}$ & 0.8242 & 0.4129 & 54 & 49 & $35.8 \%$ & $2.28(1.02,5.12)$ & & - & \\
\hline Petrovic et al (2002) $)^{69}$ & 1.3863 & 0.7159 & 20 & 20 & $18.2 \%$ & $4.00(0.98,16.27)$ & & & \\
\hline Subtotal $(95 \% \mathrm{Cl})$ & & & 139 & 129 & $100.0 \%$ & $3.90(1.94,7.82)$ & & & \\
\hline \multirow{2}{*}{\multicolumn{9}{|c|}{$\begin{array}{l}\text { Heterogeneity: } \tau^{2}=0.18 ; \chi^{2}=4.69, \text { d.f. }=3(P=0.20) ; I^{2}=36 \% \\
\text { Test for overall effect: } Z=3.83(P=0.0001)\end{array}$}} & \\
\hline & & & & & & & & & \\
\hline \multicolumn{9}{|c|}{ Withdrawal with pharmacotherapy } & \\
\hline Di Costanzo \& Rovea (1992) ${ }^{65}$ & 1.3863 & 0.9014 & 18 & 18 & $100.0 \%$ & $4.00(0.68,23.41)$ & & & \\
\hline Subtotal $(95 \% \mathrm{Cl})$ & & & 18 & 18 & $100.0 \%$ & $4.00(0.68,23.41)$ & & & \\
\hline \multicolumn{10}{|l|}{ Heterogeneity: not applicable } \\
\hline \multicolumn{10}{|c|}{ Test for overall effect: $Z=1.54(P=0.12)$} \\
\hline & & & & & & 0.01 & 0.1 & 0 & 100 \\
\hline
\end{tabular}

Fig. 3 Pooled odds ratios in relation to not using benzodiazepines in studies aimed at withdrawal from these drugs at 0.5-3 months follow-up.

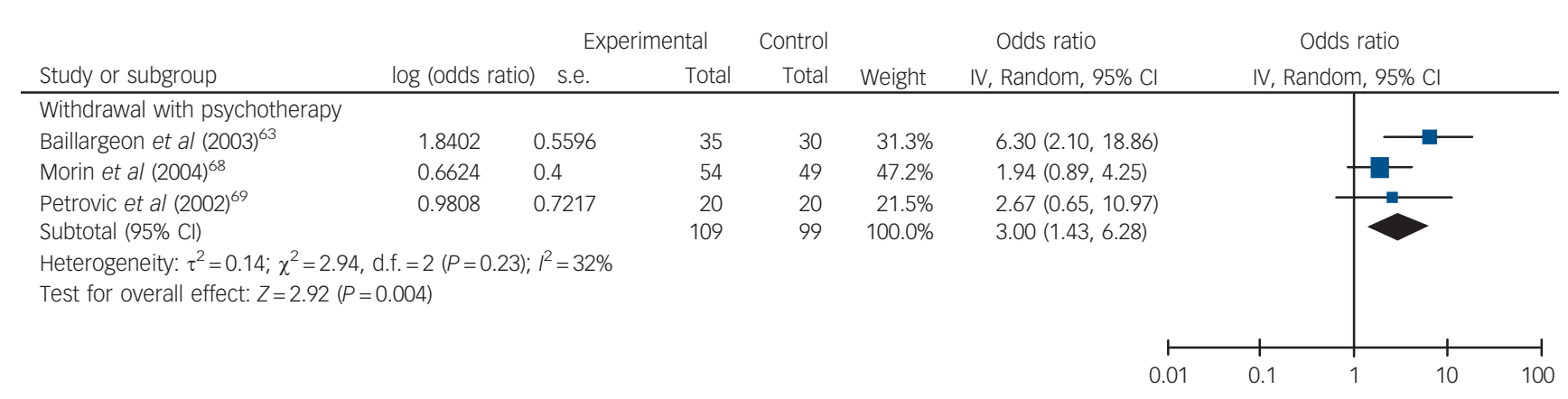

Fig. 4 Pooled odds ratios in relation to not using benzodiazepines in studies aimed at withdrawal from these drugs at 12 months follow-up. 


\section{Subgroup and meta-regression analyses}

At post-intervention assessment significant differences were found in subgroup analyses comparing type of intervention $(P=0.002)$ and underlying pathology $(P=0.005)$ but not setting $(P=0.09$; Table DS4). Similarly, meta-regression analyses revealed a significant association between effect sizes and type of intervention $(P=0.007)$ and underlying pathology $(P=0.01)$, but not setting $(P=0.21$; Table 1$)$. Higher odds of not using benzodiazepines were found in studies employing supervised withdrawal and psychotherapy compared with withdrawal and a prescribing intervention or supervised withdrawal with pharmacotherapy, and also in studies aimed at people with insomnia compared with anxiety, mixed or unreported diagnoses. A joint test of covariates in a multivariate meta-regression analysis showed there was a significant association of at least one of these variables with effect sizes $(P=0.02)$. However, neither variable was independently associated with between-study heterogeneity after adjusting for multiple comparisons ( $P=0.20$ for intervention; $P=0.54$ for underlying pathology; Table 2).

At $0.5-3$ months follow-up it was not possible to detect any significant between-group differences in mean effect sizes in subgroup analyses $(P=0.98$ for type of intervention, underlying pathology and setting; Table DS4). A limited number of studies precluded subgroup analysis at 12 months follow-up and meta-regression analyses at $0.5-3$ months and 12 months follow-up.

\section{Sensitivity analyses and publication bias}

Sensitivity analyses revealed the same pattern of results at all time points (online Table DS5). There was no evidence of publication bias in any analysis bar one: a subgroup analysis of studies in care home and in-patient settings at post-intervention $(P=0.003)$. When data were adjusted using a trim and fill method, reduced odds of not using benzodiazepines were found in these settings, although this was not significant $(\mathrm{OR}=0.81,95 \%$ CI $0.39-1.66$, $z=-0.59, P=0.56$ ).

\section{Meta-analysis: prescribing interventions}

\section{odds of not using benzodiazepines}

At post-intervention three studies did not appear to adjust for clustering at the individual level for the outcome of interest. ${ }^{74,75,78}$ All used care homes as the unit of randomisation and so an ICC of 0.057 was used to estimate the design effect as this has been reported in a study of benzodiazepine prescribing in care homes. ${ }^{80}$ As shown in Fig. 5, significantly higher odds of not using benzodiazepines were found with multifaceted prescribing interventions. The average effect of treatment at post-intervention was to make the odds of not using benzodiazepines 1.37 times higher for multifaceted interventions (95\% CI 1.10-1.72, $P=0.006, \mathrm{NNT}=15)$ compared with control interventions. In contrast, the odds were lower for single-faceted interventions in comparison with control interventions $(\mathrm{OR}=0.87,95 \% \mathrm{CI}$ $1.07-1.73, P=0.27, \mathrm{NNT}=-55)$. It was not possible to examine the longer-term effectiveness of multi- and single-faceted prescribing interventions as no study assessed outcomes at $0.5-3$ months or 12 months follow-up.

\section{Subgroup and meta-regression analyses}

Significant differences were found in subgroup analyses comparing type of intervention $(P=0.007)$ but not setting $(P=0.10$; see Table DS4). It was not possible to examine subgroup differences with respect to underlying pathology as studies rarely reported these data. Meta-regression analyses similarly showed a significant association between effect sizes and type of intervention $(P=0.04)$, but not setting $(P=0.20$; Table 1$)$. The odds of not

Table 1 Random effects univariate meta-regression analyses at post-intervention

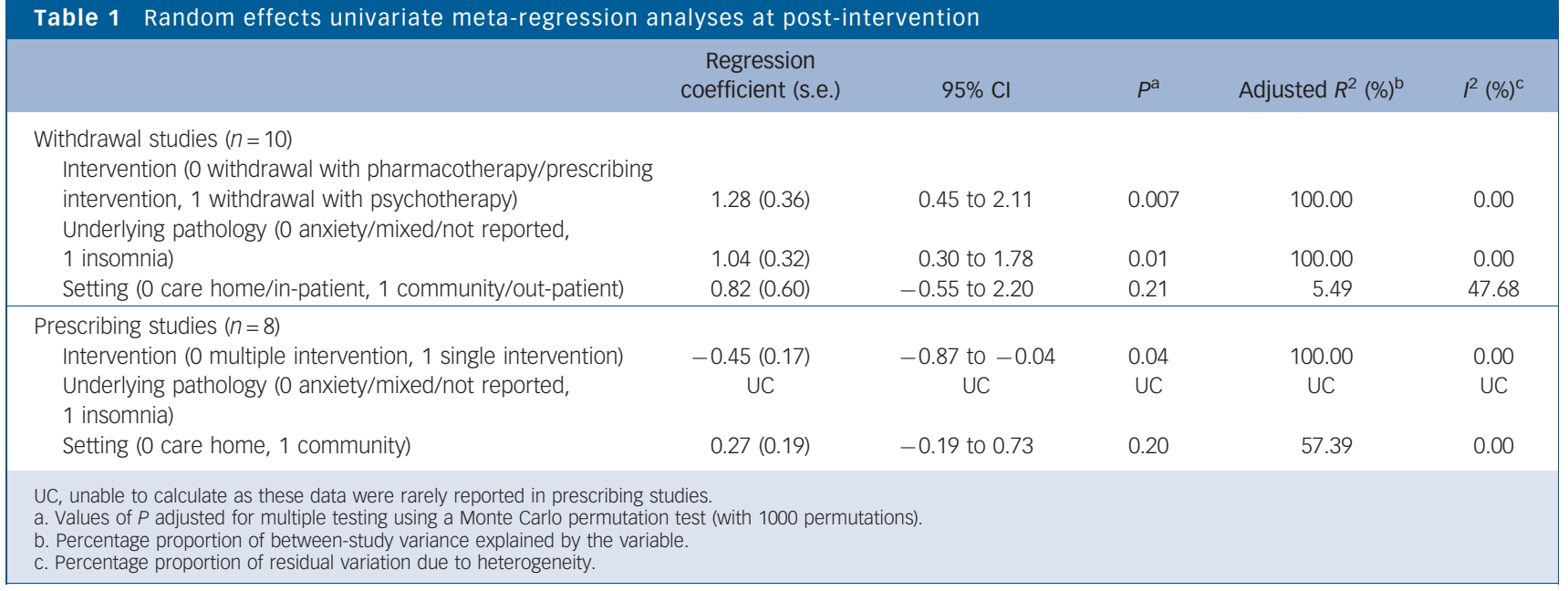

Table 2 Random-effects multivariate meta-regression analyses at post-intervention

\begin{tabular}{|c|c|c|c|c|c|}
\hline & Adjusted $P^{a}$ & $F$ & $P$ for joint test ${ }^{b}$ & Adjusted $R^{2}(\%)^{\mathrm{C}}$ & $P^{2}(\%)^{\mathrm{d}}$ \\
\hline \multicolumn{6}{|l|}{ Withdrawal studies $(n=10)$} \\
\hline Intervention & 0.20 & 6.87 & 0.02 & 100.00 & 0.00 \\
\hline Underlying pathology & 0.54 & & & & \\
\hline \multicolumn{6}{|c|}{$\begin{array}{l}\text { a. Values adjusted for multiple testing using a Monte Carlo permutation test (with } 1000 \text { permutations). } \\
\text { b. Joint test with Knapp-Hartung modification of all covariates significant at } P<0.05 \text { in univariate meta-regression. } \\
\text { c. Percentage proportion of between-study variance explained by the variable. } \\
\text { d. Percentage proportion of residual variation due to heterogeneity. }\end{array}$} \\
\hline
\end{tabular}




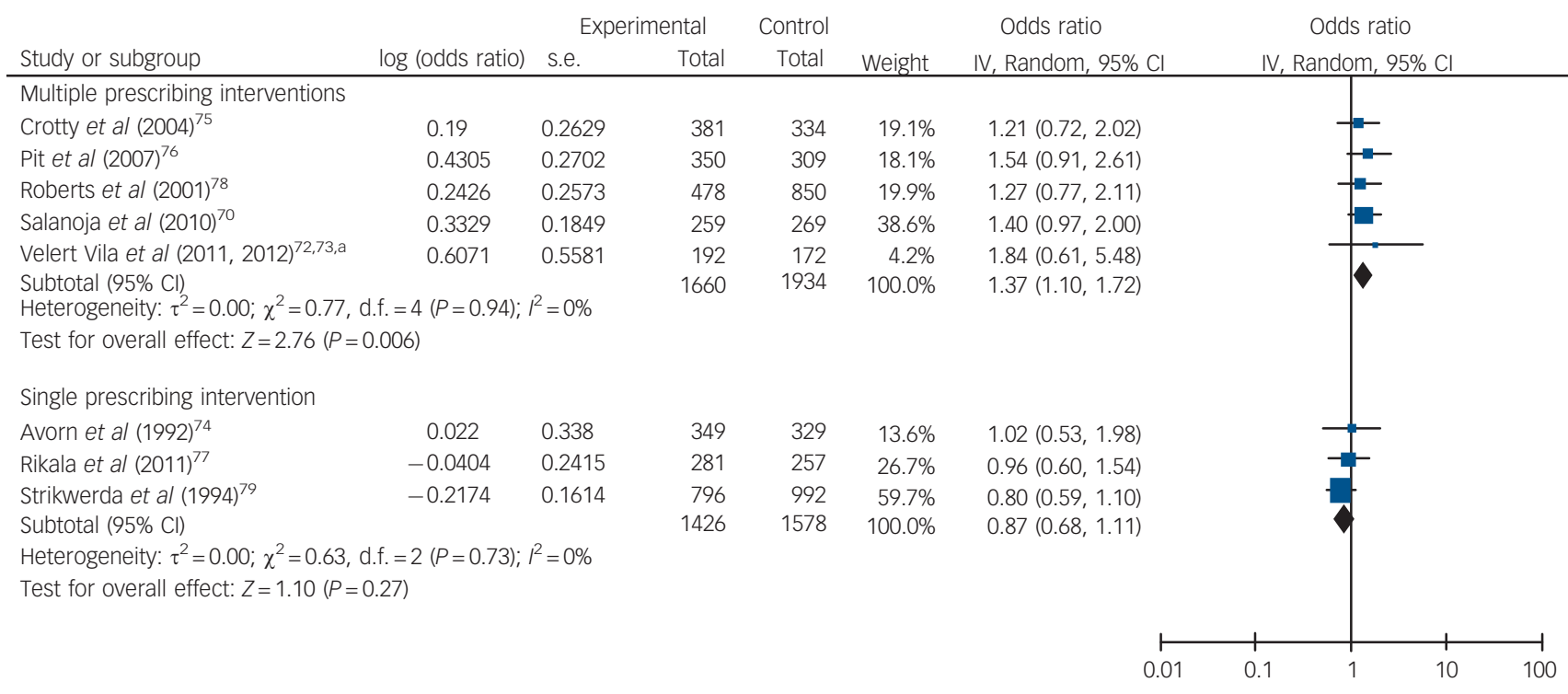

Fig. 5 Pooled odds ratios in relation to not using benzodiazepines in studies aimed at changing benzodiazepine prescribing (and hence use of these drugs) at post-intervention.

a. Those leaving this study were assumed to have continued using benzodiazepines (for all other studies this was not possible to calculate).

using benzodiazepines were higher for multifaceted prescribing interventions (in comparison with control interventions) than for single-faceted interventions (in comparison with control interventions).

\section{Sensitivity analyses and publication bias}

The same pattern of results was found in sensitivity analyses at post-intervention (Table DS5). There was no evidence of publication bias.

\section{Discussion}

The odds of not using benzodiazepines were significantly higher for supervised withdrawal with psychotherapy in comparison with control interventions in the short term. Number needed to treat analyses indicated that 1 additional person would not use benzodiazepines for every 3 receiving supervised withdrawal with psychotherapy rather than the control intervention. The beneficial effects of supervised withdrawal with psychotherapy were maintained at 0.5-3 months and 12 months follow-up. However, evidence was weakened by non-significant but moderate heterogeneity in effect sizes: $95 \%$ prediction intervals suggested that supervised withdrawal with psychotherapy might not always be effective in individual settings at these two follow-up points.

Few studies examined both short-term and long-term effectiveness of withdrawal with a prescribing intervention. In two studies there was evidence that the odds of not using benzodiazepines were significantly higher for withdrawal with a prescribing intervention in comparison with control interventions in the short term, although the magnitude of this effect was small, as demonstrated by an NNT of 13. These results should be interpreted with caution given the small number of studies included in the analysis. No conclusion could be drawn with respect to long-term effects owing to a lack of studies. There was little evidence to support the use of supervised withdrawal with pharmacotherapy compared with control interventions in the short term. The NNT analyses indicated that 1 additional person would not use benzodiazepines for every 20 receiving supervised withdrawal with pharmacotherapy rather than the control intervention at post-intervention. Again, it was not possible to draw any conclusion with respect to longer-term effects owing to a lack of studies. There was evidence that multifaceted interventions aimed at changing prescribing (and hence benzodiazepine use) were effective at significantly increasing the odds of not using benzodiazepines in comparison with control interventions in the short term. However, the magnitude of this effect was small. The NNT analyses indicated that 1 additional person would not use benzodiazepines for every 15 treated with multifaceted interventions rather than the control intervention at post-treatment. It was not possible to detect evidence that single-faceted interventions increased the odds of not using benzodiazepines compared with control interventions. However, this may in part be due to the smaller number of available studies, thus these results should be interpreted with caution. No study examined longer-term effects of interventions aimed at changing prescribing.

\section{Comparisons with other studies}

The finding that supervised withdrawal with psychotherapy was more effective than other withdrawal interventions in the short term is consistent with previous meta-analyses. ${ }^{18,20}$ Evidence that withdrawal with a prescribing intervention may also be effective in reducing benzodiazepine use, albeit to a small degree, is also consistent with other meta-analyses. ${ }^{18-20}$ The lack of evidence to support the use of supervised withdrawal with pharmacotherapy compared with control interventions is in accord with one study, ${ }^{18}$ but not another. ${ }^{20}$ However, the latter study only reported significantly higher discontinuation rates for the augmentation of withdrawal with imipramine, but not other pharmaceutical agents, in comparison with control interventions. No study in the current meta-analysis examined withdrawal with imipramine. Multifaceted interventions aimed at changing benzodiazepine prescribing were found to be more effective at increasing the odds of not using benzodiazepines in comparison with single-faceted interventions. This is consistent with the conclusions of a previous non-systematic review. ${ }^{25}$ The fact that multifaceted prescribing interventions comprised education and medication review in 
four out of five studies is also in accord with previous reviews which concluded that these components of the interventions were the most beneficial for reducing drug use. ${ }^{23,24}$

Although direct comparisons were not made between studies of younger $v$. older people (as this was beyond the scope of this review), similarities were found between our findings in older people and those of previous meta-analyses in adults of any age. These similarities suggest that interventions aimed at reducing benzodiazepine use (through withdrawal or benzodiazepine prescribing strategies) may be as applicable to older people as they are in wider populations. The exception may be interventions involving supervised withdrawal with pharmacotherapy, which may in part be related to the type of pharmaceutical compound used.

\section{Limitations of the study}

First, the small number of studies (particularly at follow-up) limited the strength and reliability of the conclusions that could be drawn about the effectiveness of interventions for reducing benzodiazepine use. Second, subgroup and meta-regression analyses examined whether any between-study heterogeneity could be explained by the type of intervention, underlying pathology or setting. It was not possible to examine the moderating effect of underlying pathology on effect sizes in prescribing studies as this was rarely reported. In addition, although significant associations were found between effect sizes and type of intervention and underlying pathology in some analyses, the possibility that another moderating variable might have been responsible for these differences cannot be ruled out. Furthermore, results should be interpreted with caution owing to small study numbers and the fact that associations across studies may not be the same as those within studies. ${ }^{81}$ Third, although there was evidence of publication bias in one subgroup analysis alone, the inclusion of unpublished data or data from controlled trials (rather than solely from RCTs) may have altered pooled odds ratios and hence conclusions drawn in the current meta-analysis. Fourth, two studies examined withdrawal within the context of a multifaceted prescribing intervention; consequently, the exact mechanism of change or 'active ingredient' in these studies is unknown. Finally, a conservative approach to data extraction was adopted following a previous meta-analysis, ${ }^{18}$ whereby all patients who left the study were assumed to have continued using benzodiazepines. This assumption might not always have applied to this group, and so results may be overly conservative. In addition, it was not possible to use this approach for all studies as some did not report withdrawal rates specifically for patients using benzodiazepine. The majority of studies in which this conservative approach could not be used were those that targeted changing benzodiazepine prescribing. As a result, different conclusions may have been drawn about the effectiveness of multi- $v$. single-faceted prescribing interventions for reducing benzodiazepine use had this occurred.

\section{Clinical and research implications}

Despite these limitations, there are a number of clinical and research implications of our findings. In two studies examining benzodiazepine dependence in older people, $43 \%$ of those taking these drugs considered themselves to be addicted, ${ }^{10}$ whereas $9.5 \%$ were found to meet DSM-IV criteria for benzodiazepine dependence. ${ }^{82}$ In the current meta-analysis, all bar one of the psychotherapy studies reported addressing coping with dependency/withdrawal symptoms, together with underlying pathology (e.g. insomnia or anxiety). Few details were provided about the 'psychological consulting' intervention, but it is likely that this addressed similar issues. In contrast, these factors were not reported to have been addressed in withdrawal with pharmacotherapy studies. It is not possible from this meta-analysis to determine whether beneficial treatment outcomes were attributable to addressing the interpretation and experience of withdrawal symptoms, underlying pathology, or both. However, an RCT conducted in younger and older adults reported no difference in benzodiazepine discontinuation rates between gradual withdrawal alone and gradual withdrawal plus CBT specifically targeted at dependency/withdrawal rather than underlying pathology. ${ }^{37,83}$ This suggests that the key mechanism of change in psychotherapy interventions might be targeting underlying pathology rather than dependency and withdrawal issues.

In addition to examining potential mechanisms of change further, future studies could explore possible moderators of treatment response in older people. In particular, the results of the subgroup and meta-regression analyses suggest that type of intervention and underlying pathology could be examined. The results further suggest that a factorial design nested within an RCT, such as underlying pathology (insomnia $v$. anxiety) by type of intervention (withdrawal with psychotherapy $v$. withdrawal with pharmacotherapy), might be invaluable in addressing this issue. The findings of this meta-analysis also highlight the need for more studies aimed at establishing the long-term effectiveness of specific interventions for reducing benzodiazepine use in older people. In addition, studies could explore withdrawal with other pharmaceutical agents such as imipramine, together with optimal benzodiazepine tapering schedules, given that little is known about this in adults of any age, ${ }^{84,85}$ let alone older people. Furthermore, they could examine economic evaluations of augmented withdrawal and multifaceted prescribing interventions to determine whether improvements in clinical outcomes are outweighed by the costs of implementing such interventions in clinical practice. Although relatively small increases in cost-effectiveness have been reported for withdrawal alone $v$. withdrawal with psychotherapy in an RCT involving adults of any age, ${ }^{86}$ little is known about this in older people.

Finally, the results of the meta-analysis imply that a multistrategy approach incorporating both supervised withdrawal with psychotherapy and multifaceted prescribing interventions, with 'buy-in' from both prescribers and patients, as highlighted in a recent review, ${ }^{27,87}$ could be most beneficial for reducing benzodiazepine use in older people. Two studies in the current metaanalysis examined withdrawal alone combined with multifaceted prescribing interventions..$^{70,72,73}$ However, no study to date has explored whether combining supervised withdrawal with psychotherapy (targeted at patients) and multifaceted prescribing interventions such as education, medication review and audit/ prescribing feedback (targeted at physicians, healthcare staff and patients) could improve clinical outcomes in older people in comparison with either approach alone. With benzodiazepine prevalence rates remaining high in older people, there is clearly a need for further exploration of combinations of interventions that are most effective at reducing benzodiazepine use in this population.

\section{Clinical recommendations}

Evidence reviewed here, albeit limited, suggests that a number of strategies might be beneficial in assisting older people to withdraw from benzodiazepines: first, medication review and consultation, together with provision of a withdrawal schedule and education about benzodiazepine use (for both those taking and those 
prescribing benzodiazepines); and second, provision of a supervised withdrawal schedule augmented with psychotherapy (mainly aimed at addressing underlying pathology). Although higher odds of not using benzodiazepines were found with the latter strategy, pragmatic reasons (such as access to psychotherapy) may mean that the former strategy is initially preferred within a stepped care approach.

Rebecca L. Gould, PhD, DClinPsy, King's College London, Department of Old Age Psychiatry, Institute of Psychiatry, London; Mark C. Coulson, PhD, Department of Psychology, School of Health and Education, Middlesex University, Hendon; Natasha Patel, MBBS, Elizabeth Highton-Williamson, BSC, Robert J. Howard, MD, King's College London, Department of Old Age Psychiatry, Institute of Psychiatry, London, UK

Correspondence: Dr Rebecca Gould, Department of Old Age Psychiatry,

Institute of Psychiatry, De Crespigny Park, London SE5 8AF, UK. Email:

rebecca.gould@kcl.ac.uk.

First received 8 Jan 2013, final revision 24 Jul 2013, accepted 5 Sep 2013

\section{Funding}

This research was supported by the Mental Health of Older Adults and Dementia Clinical Academic Group within King's Health Partners Academic Health Sciences Centre at the Institute of Psychiatry, King's College London and the South London and Maudsley National Institute of Psychiatry, King's Coll
Health Service Foundation Trust.

\section{Acknowledgements}

The authors would like to express their gratitude and thanks to all those who responded to data queries and to three anonymous reviewers for their helpful comments.

\section{References}

1 Beers MH, Ouslander JG, Rollingher I, Reuben DB, Brooks J, Beck JC. Explicit criteria for determining inappropriate medication use in nursing-home residents. Arch Intern Med 1991; 151: 1825-32.

2 Fick DM, Cooper JW, Wade WE, Waller JL, Maclean JR, Beers MH. Updating the Beers criteria for potentially inappropriate medication use in older adults: results of a US consensus panel of experts. Arch Intern Med 2003; 163: 2716-24.

3 Ashton $\mathrm{CH}$. The Ashton Manual. Benzodiazepines: How They Work and How to Withdraw. 2002. http://www.benzo.org.uk/manual/.

4 Department of Health. Benzodiazepines warning. In Chief Medical Officer's Update 37, Patient Safety. Department of Health, 2004.

5 Buffett-Jerrott SE, Stewart SH. Cognitive and sedative effects of benzodiazepine use. Curr Pharm Des 2002; 8: 45-58.

6 Hill KD, Wee R. Psychotropic drug-induced falls in older people: a review of interventions aimed at reducing the problem. Drugs Aging 2012; 29: 15-30.

7 Cumming RG, Le Couteur DG. Benzodiazepines and risk of hip fractures in older people: a review of the evidence. CNS Drugs 2003; 17: 825-37.

8 Meuleners LB, Duke J, Lee AH, Palamara P, Hildebrand J, Ng JQ. Psychoactive medications and crash involvement requiring hospitalization for older drivers: a population-based study. J Am Geriatr Soc 2011; 59: 1575-80.

9 Clegg A, Young JB. Which medications to avoid in people at risk of delirium: a systematic review. Age Ageing 2011; 40: 23-9.

10 Voyer P, Preville M, Martin LS, Roussel ME, Beland SG, Berbiche D. Factors associated with self-rated benzodiazepine addiction among community-dwelling seniors. J Addict Nurs 2011; 22: 46-56.

11 American Geriatrics Society Beers Criteria Update Expert Panel. American Geriatrics Society updated Beers Criteria for potentially inappropriate medication use in older adults. J Am Geriatr Soc 2012; 60: 616-31.

12 Johnell K, Fastbom J. The use of benzodiazepines and related drugs amongst older people in Sweden: associated factors and concomitant use of other psychotropics. Int J Geriatr Psychiatry 2009; 24: 731-8.

13 Dionne PA, Vasiliadis HM, Latimer E, Berbiche D, Preville M. Economic impact of inappropriate benzodiazepine prescribing and related drug interactions among elderly persons. Psychiatr Serv 2013; 64: 331-8.

14 Martinsson G, Fagerberg I, Wiklund-Gustin L, Lindholm C. Specialist prescribing of psychotropic drugs to older persons in Sweden a register-based study of 188024 older persons. BMC Psychiatry 2012 12: 197
15 Preville M, Vasiliadis HM, Bosse C, Dionne PA, Voyer P, Brassard J, et al. Pattern of psychotropic drug use among older adults having a depression or an anxiety disorder: Results from the longitudinal ESA study. Can J Psychiatry 2011; 56: 348-57

16 Lasserre A, Younes N, Blanchon T, Cantegreil-Kallen I, Passerieux C, Thomas G, et al. Psychotropic drug use among older people in genera practice: discrepancies between opinion and practice. Br J Gen Pract 2010; 60: $156-62$

17 Russell J, Lader M. Guidelines for the Prevention and Treatment of Benzodiazepine Dependence. Mental Health Foundation, 1993.

18 Parr JM, Kavanagh DJ, Cahill L, Mitchell G, Young RM. Effectiveness of current treatment approaches for benzodiazepine discontinuation: a meta-analysis. Addiction 2008; 104: 13-24.

19 Mugunthan K, McGuire T, Glasziou P. Minimal interventions to decrease long-term use of benzodiazepines in primary care: a systematic review and meta-analysis. Br J Gen Pract 2011; 61: 573-8.

20 Oude Voshaar RC, Couvée JE, Van Balkom AJLM, Mulder PGH, Zitman FG. Strategies for discontinuing long-term benzodiazepine use. Meta-analysis. Br J Psychiatry 2006; 189: 213-20.

21 Denis C, Fatseas M, Lavie E, Auriacombe M. Pharmacological interventions for benzodiazepine mono-dependence management in outpatient settings. Cochrane Database Syst Rev 2006; 3: CD005194.

22 Milton JC, Hill-Smith I, Jackson SHD. Prescribing for older people. BMJ 2008; 336: $606-9$.

23 Forsetlund L, Eike MC, Gjerberg E, Vist GE. Effect of interventions to reduce potentially inappropriate use of drugs in nursing homes: a systematic review of randomised controlled trials. BMC Geriatr 2011; 11: 16.

24 Nishtala PS, McLachlan AJ, Bell JS, Chen TF. Psychotropic prescribing in long-term care facilities: impact of medication reviews and educational interventions. Am J Geriatr Psychiatry 2008; 16: 621-32.

25 Smith AJ, Tett SE. Improving the use of benzodiazepines - is it possible? A non-systematic review of interventions tried in the last 20 years. BMC Health Serv Res 2010; 10: 321

26 Marcum ZA, Handler SM, Wright R, Hanlon JT. Interventions to improve suboptimal prescribing in nursing homes: a narrative review. Am J Geriatr Pharmacother 2010; 8: 183-200.

27 Ostini R, Jackson C, Hegney D, Tett SE. How is medication prescribing ceased? A systematic review. Med Care 2011; 49: 24-36.

28 Cantopher T, Olivieri S, Cleave N, Edwards JG. Chronic benzodiazepine dependence. A comparative study of abrupt withdrawal under propranolol cover versus gradual withdrawal. Br J Psychiatry 1990; 156: 406-11.

29 Cormack MA, Sweeney KG, Hughesjones H, Foot GA. Evaluation of an easy, cost-effective strategy for cutting benzodiazepine use in general practice. Br J Gen Pract 1994; 44: 5-8.

30 Schweizer E, Rickels K, De Martinis N, Case G, Garcia-Espana F. The effect of personality on withdrawal severity and taper outcome in benzodiazepine dependent patients. Psychol Med 1998; 28: 713-20.

31 Schweizer E, Case WG, Rickels K. Benzodiazepine dependence and withdrawal in elderly patients. Am J Psychiatry 1989; 146: 529-31.

32 Ashton $\mathrm{H}$. Benzodiazepine withdrawal - outcome in 50 patients. $\mathrm{Br} J$ Addict 1987: 82: 665-71.

33 Holden JD, Hughes IM, Tree A. Benzodiazepine prescribing and withdrawal for 3234 patients in 15 general practices. Fam Pract 1994; 11: 358-62.

34 Lemoine P, Kermadi I, Garcia-Acosta S, Garay RP, Dib M. Double-blind comparative study of cyamemazine vs. bromazepam in the benzodiazepine withdrawal syndrome. Prog Neuropsychopharmacol Biol Psychiatry 2006; 30 131-7.

35 Rickels K, Case WG, Schweizer E, Garciaespana F, Fridman R. Long-term benzodiazepine users 3 years after participation in a discontinuation program. Am J Psychiatry 1991; 148: 757-61.

36 Gorgels W, Oude Voshaar RC, Mol AJ, van de Lisdonk EH, van Balkom A, Breteler MHM, et al. Predictors of discontinuation of benzodiazepine prescription after sending a letter to long-term benzodiazepine users in family practice. Fam Pract 2006; 23: 65-72.

37 Oude Voshaar RC, Gorgels WJMJ, Mol AJJ, Van Balkom AJLM, Van de Lisdonk EH, Breteler MHM, et al. Tapering off long-term benzodiazepine use with or without group cognitive-behavioura therapy: three-condition, randomised controlled trial. Br J Psychiatry 2003; 182: 498-504.

38 Schweizer E, Rickels K, Case WG, Greenblatt DJ. Long-term therapeutic use of benzodiazepines II. Effects of gradual taper. Arch Gen Psychiatry 1990; 47 908-15.

39 Smith DH, Christensen DB, Stergachis A, Holmes G. A randomized controlled trial of a drug use review intervention for sedative hypnotic medications. Med Care 1998; 36: 1013-21. 
40 Stewart R, Niessen WJM, Broer J, Snijders TAB, Haaijer-Ruskamp FM, Jong BMD. General practitioners reduced benzodiazepine prescriptions in an intervention study: a multilevel application. J Clin Epidemiol 2007; 60: 1076-84.

41 Ten Wolde GB, Dijkstra A, Van Empelen P, van den Hout W, Neven AK, Zitman F. Long-term effectiveness of computer-generated tailored patient education on benzodiazepines: a randomized controlled trial. Addiction 2008 103: $662-70$.

42 Tyrer $\mathrm{P}$, Owen R, Dawling S. Gradual withdrawal of diazepam after long-term therapy. Lancet 1983; 1: 1402-6.

43 Foy A, Drinkwater V, March S, Mearrick P. Confusion after admission to hospital in elderly patients using benzodiazepines. BMJ 1986; 293: 1072.

44 Cormack MA, Sinnott A. Psychological alternatives to long-term benzodiazepine use. J $R$ Coll Gen Pract 1983; 33: 279-81.

45 Covin R, Ouimet AJ, Seeds PM, Dozois DJ. A meta-analysis of CBT for pathological worry among clients with GAD. J Anxiety Disord 2008; 22 108-16.

46 Department of Health. National Service Framework for Older People. Department of Health, 2001.

47 United Nations. World Economic and Social Survey. UN, 2007 (http://www.un.org/esa/policy/wess/wess2007files/chap1.pdf).

48 Woods JH, Katz JL, Winger GD. Benzodiazepines: use, abuse, and consequences. Pharmacol Rev 1992; 44: 151-347.

49 Higgins JPT, Altman DG, Sterne JAC. Chapter 8: Assessing risk of bias in included studies. In Cochrane Handbook for Systematic Reviews of Interventions (Version 5.1.0) (eds JPT Higgins, S Green). The Cochrane Collaboration, 2011.

50 Higgins JPT, Green S. Cochrane Handbook for Systematic Reviews of Interventions, version 5.1.0. Cochrane Collaboration, 2011 (http:// www.cochrane-handbook.org).

51 Donner A, Klar N. Issues in the meta-analysis of cluster randomized trials. Stat Med 2002; 21: 2971-80.

52 Elbourne DR, Altman DG, Higgins JPT, Curtin F, Worthington HV, Vail A. Metaanalyses involving cross-over trials: methodological issues. Int J Epidemiol 2002; 31: 140-9.

53 StataCorp. Stata Statistical Software: Release 11.2. StataCorp, 2011.

54 Cochrane Collaboration. Review Manager (RevMan) [computer program]. Version 5.1. Nordic Cochrane Centre, Cochrane Collaboration, 2012.

55 Higgins JPT, Thompson SG, Deeks JJ, Altman DG. Measuring inconsistency in meta-analyses. BMJ 2003; 327: 557-60.

56 Riley RD, Higgins JPT, Deeks JJ. Interpretation of random effects metaanalyses. BMJ 2011; 342: 549.

57 Egger M, Smith GD, Schneider M, Minder C. Bias in meta-analysis detected by a simple, graphical test. BMJ 1997; 315: 629-34.

58 Duval S, Tweedie R. Trim and fill: a simple funnel-plot-based method of testing and adjusting for publication bias in meta-analysis. Biometrics 2000; 56: 455-63.

59 Borenstein M, Hedges LV, Higgins JPT, Rothstein HR. Introduction to Meta-Analysis. Wiley, 2009

60 Nakanishi Y, Toyoizumi S, Nakajima A, Hamada C. Subgroup analysis in meta-analysis: a comparison of different methods. Jpn J Pharmacoepidemiol 2007; 12: 13-24.

61 Higgins JPT, Thompson SG. Controlling the risk of spurious findings from meta-regression. Stat Med 2004; 23: 1663-82.

62 Moher D, Liberati A, Tetzlaff J, Altman DG. Preferred reporting items for systematic reviews and meta-analyses: the PRISMA statement. BMJ 2009; 339: 2535.

63 Baillargeon L, Landreville P, verreault R, Beauchemin JP, Gregoire JP, Morin $\mathrm{CM}$. Discontinuation of benzodiazepines among older insomniac adults treated with cognitive-behavioural therapy combined with gradual tapering: a randomized trial. CMAJ 2003; 169: 1015-20.

64 Cardinali DP, Gvozdenovich E, Kaplan MR, Fainstein I, Shifis HA, Lloret SP, et al. A double blind-placebo controlled study on melatonin efficacy to reduce anxiolytic benzodiazepine use in the elderly. Neuro Endocrinol Lett 2002; 23: 55-60.

65 Di Costanzo E, Rovea A. The prophylaxis of benzodiazepine withdrawal syndrome in the elderly: the effectiveness of carbamazepine. Double-blind study vs. placebo. Minerva Psichiatr 1992; 33: 301-4.

66 Giblin MJ, Clift AD. Sleep without drugs. J R Coll Gen Pract 1983; 33: 628-33.
67 Habraken H, Soenen K, Blondeel L, VanElsen J, Bourda J, Coppens E, et al Gradual withdrawal from benzodiazepines in residents of homes for the elderly: experience and suggestions for future research. Eur J Clin Pharmacol 1997; 51: 355-8.

68 Morin CM, Bastien C, Guay B, Radouco-Thomas M, Leblanc J, Vallieres A. Randomized clinical trial of supervised tapering and cognitive behavior therapy to facilitate benzodiazepine discontinuation in older adults with chronic insomnia. Am J Psychiatry 2004; 161: 332-42.

69 Petrovic M, Pevernagie D, Mariman A, Van Maele G, Afschrift M. Fast withdrawal from benzodiazepines in geriatric inpatients: a randomised double-blind, placebo-controlled trial. Eur J Clin Pharmacol 2002; 57: 759-64.

70 Salonoja M, Salminen M, Aarnio P, Vahlberg T, Kivela SL. One-time counselling decreases the use of benzodiazepines and related drugs among community-dwelling older persons. Age Ageing 2010; 39: 313-9.

71 Tham TCK, Brown H, Taggart HM. Temazepam withdrawal in elderly hospitalized patients - a double-blind randomized trial comparing abrupt versus gradual withdrawal. Ir J Med Sci 1989; 158: 294-9.

72 Velert Vila J. Intervención Farmacéutica para la Adecuación de la Prescripción de Benzodiazepinas en Pacientes Mayores [Pharmaceutical Intervention for the Suitability of Benzodiazepine Prescription in Older Patients]. Cardinal Herrera University, 2011.

73 Velert Vila J, del Mar Velert Vila M, Salar Ibanez L, Avellana Zaragoza JA Moreno Royo L. Adecuación de la utilización de benzodiazepinas en ancianos desde la oficina de farmacia. Un estudio de colaboración médicofarmacéutico [Suitability of the use of benzodiazepines prescribed by the pharmacist in the elderly. A doctor-pharmacist collaboration study]. Aten Primaria 2012; 44: 402-10.

74 Avorn J, Soumerai SB, Everitt DE, Rossdegnan D, Beers MH, Sherman D, et al. A randomized trial of a program to reduce the use of psychoactive drugs in nursing homes. N Engl J Med 1992; 327: 168-73.

75 Crotty M, Whitehead C, Rowett D, Halbert J, Weller D, Finucane P, et al. An outreach intervention to implement evidence based practice in residential care: a randomized controlled trial. BMC Health Serv Res 2004; 4: 6.

76 Pit SW, Byles JE, Henry DA, Holt L, Hansen V, Bowman DA. A Quality Use of Medicines program for general practitioners and older people: a cluster randomised controlled trial. Med J Aust 2007; 187: 23-30.

77 Rikala M, Korhonen MJ, Sulkava R, Hartikainen S. The effects of medication assessment on psychotropic drug use in the community-dwelling elderly. Int Psychogeriatr 2011; 23: 473-84.

78 Roberts MS, Stokes JA, King MA, Lynne TA, Purdie DM, Glasziou PP, et al. Outcomes of a randomized controlled trial of a clinical pharmacy intervention in 52 nursing homes. Br J Clin Pharmacol 2001; 51: 257-65.

79 Strikwerda P, Bootsma-de Langen AM, Berghuis F, Meyboom-de Jong B. Drug therapy in a nursing home; favorable effect of feedback by the pharmacist on family physician's prescribing behavior. Ned Tijdschr Geneeskd 1994; 138: 1770-4.

80 Oborne CA, Hooper R, Swift CG, Jackson SHD. Explicit, evidence-based criteria to assess the quality of prescribing to elderly nursing home residents. Age Ageing 2003; 32: 102-8.

81 Thompson SG, Higgins JPT. How should meta-regression analyses be undertaken and interpreted? Stat Med 2002; 21: 1559-73.

82 Voyer P, Preville M, Cohen D, Berbiche D, Beland SG. The prevalence of benzodiazepine dependence among community-dwelling older adult users in Quebec according to typical and atypical criteria. Can J Aging 2010; 29: 205-13.

83 Oude Voshaar RC, Gorgels WJMJ, Mol AJJ, Van Balkom AJLM, Mulder J, Van de Lisdonk $\mathrm{EH}$, et al. Long-term outcome of two forms of randomised benzodiazepine discontinuation. Br J Psychiatry 2006; 188: 188-9.

84 Dell'osso B, Lader M. Do benzodiazepines still deserve a major role in the treatment of psychiatric disorders? A critical reappraisal. Eur Psychiatry 2013; 28: 7-20.

85 Lader M, Tylee A, Donoghue J. Withdrawing benzodiazepines in primary care. CNS Drugs 2009; 23: 19-34.

86 Oude Voshaar RC, Krabbe PFM, Gorgels WJMJ, Adang EM, van Balkom AJ, van de Lisdonk EH, et al. Tapering off benzodiazepines in long-term users: an economic evaluation. Pharmacoeconomics 2006; 24: 683-94.

87 Ostini R, Hegney D, Jackson C, Tett SE. Knowing how to stop: ceasing prescribing when the medicine is no longer required. J Manag Care Pharm 2012; 18: 68-72. 